\title{
HOT CNO-NE CYCLES
}

(Abstract)

\author{
JEAN AUDOUZE
}

Laboratoire René Bernas, Orsay, Radio Astronomie, Observatoire de Meudon, Meudon and SEP, CEN, Saclay, France

Recent studies of the CNO-Ne cycles at temperatures higher than $10^{8} \mathrm{~K}$ are summarised. At these temperatures nuclear reactions (induced by protons or alpha particles for instance) are more rapid than the beta decay reactions. As a consequence the behaviour and the results of the nuclear processing become very different from those of the CNO cycle at lower temperature which transforms ${ }^{12} \mathrm{C}$ and ${ }^{16} \mathrm{O}$ into ${ }^{14} \mathrm{~N}$.

Audouze et al. (1973) have shown that in hydrogen rich zones the relative slowness of the beta decay reactions (favouring the beta unstable nuclei) induces large enhancements of ${ }^{13} \mathrm{C},{ }^{15} \mathrm{~N},{ }^{17} \mathrm{O}$ and ${ }^{21} \mathrm{Ne}$ at temperatures $10^{8}<T<5 \times 10^{8} \mathrm{~K}$ in time scales $1<t<10^{4} \mathrm{~s}$, while temperatures as large as $5 \times 10^{8}<T<10^{9}$ are necessary to induce overabundances of ${ }^{18} \mathrm{O}$ and ${ }^{19} \mathrm{~F}$.

The hot CNO-Ne process is likely to occur in at least two different astronomical objects: (1) Starrfield et al. (1972) have shown that explosions of novae can be triggered by hot CNO processes occurring at the surface of the white dwarf precursor of the nova. In these conditions, the matter ejected from the nova is largely enriched in ${ }^{13} \mathrm{C},{ }^{15} \mathrm{~N}$ and ${ }^{17} \mathrm{O}$. (2) Audouze and Fricke (1973) have studied the nucleosynthetic effects during the implosion-explosion of supermassive stars (of mass $\sim 10^{5} M_{\odot}$ ) and have shown that these objects, which may be present in the condensed nuclei of many galaxies, can be responsible for the formation of ${ }^{13} \mathrm{C},{ }^{15} \mathrm{~N}$ and ${ }^{17} \mathrm{O}$ provided that one per cent of the galactic matter has been processed in such massive objects.

\section{References}

Audouze, J. and Fricke, K. J.: 1973, Astrophys. J. 186, 239.

Audouze, J., Truran, J. W., and Zimmerman, B. A.: 1973, Astrophys. J. 184, 493.

Starrfield, S., Truran, J. W., Sparks, W. N., and Kutter, G. S.: 1972, Astrophy's. J. 176, 169. 